$\mathrm{isid} / \mathrm{ms} / 2002 / 29$

November 20, 2002

http://www.isid.ac.in/ statmath/eprints

\title{
Asymptotic Properties of Sums of Upper Records
}

\author{
Arup Bose \\ SREEla Gangopadhyay \\ ANISH SARKAR AND \\ Arindam Sengupta
}

Indian Statistical Institute, Delhi Centre

7, SJSS Marg, New Delhi-110 016, India 


\title{
Asymptotic Properties of Sums of Upper Records
}

\author{
Arup Bose* \\ Sreela Gangopadhyay ${ }^{\dagger}$ \\ Indian Statistical Institute, Kolkata \\ Anish Sarkar \\ Indian Statistical Institute, New Delhi \\ Arindam Sengupta ${ }^{\S}$ \\ Indian Institute of Technology, Guwahati
}

\begin{abstract}
Arnold [1] raised several questions for upper records, including characterizing all limit distributions of normalised partial sums of upper records. We provide some answers in the case when the distribution from which the samples are drawn is bounded above. When the distribution is not bounded above, we give sufficient conditions on the distribution for the properly normalized partial sums to converge to a standard normal distribution. We show that our conditions are general enough so that the examples provided by Arnold are covered by our results.
\end{abstract}

Keywords: Sums of records, infinite divisibility, asymptotic distribution.

AMS 2000 Subject Classification: 60E07, 60E10, 62E15, 62E20.

${ }^{*}$ Theoretical Statistics and Mathematics Unit, I.S.I., 203 B.T. Road, Kolkata 700108, InDIA Email: abose@isical.ac.in

${ }^{\dagger}$ Theoretical Statistics and Mathematics Unit, I.S.I., 203 B.T. Road, Kolkata 700108, InDIA Email: res9616@isical.ac.in

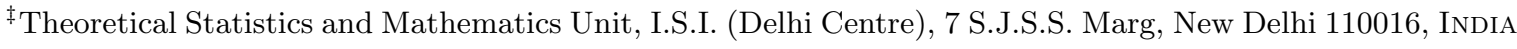
Email: anish@isid.ac.in

${ }^{\S}$ Department of Mathematics, Indian Institute of Technology Guwahati, Guwahati 781039, INDIA

Email: arindam@iitg.ernet.in 


\section{Introduction}

The history of study of asymptotic distributions of running extrema goes back to Gnedenko. In a similar spirit, Resnick [4] studied the asymptotics of record values and showed that three different limit distributions of normalised upper records are possible.

Arnold [1] considered the question of weak convergence of the sequence of partial sums of upper records. He described a few examples and raised the question of classifying all possible limit distributions.

Suppose $X_{0}$ is nonnegative and has distribution $F$. Suppose for any $n \geq 1$ the conditional distribution of $X_{n}$ given that $\left\{X_{n-1}=x\right\}$ is the same as $F$ truncated from above at $x$; that is, that of $X_{1}$ given that $X_{1} \geq x$. Then this sequence is called a sequence of upper records. Arnold [1] studied the partial sums of upper records and obtained their asymptotic distributions for some specific cases. We aim to obtain some general results which will in particular cover Arnold's examples.

Let $\left\{X_{n}: n \geq 0\right\}$ be the upper records of i.i.d. observations from a continuous distribution function $F$ supported on $[0, \infty)$. Define by $T_{n}$ the sum of first $(n+1)$ records, i.e., $T_{n}=\sum_{i=0}^{n} X_{i}$. Arnold [1] proved that

- (Exponential records) If $F(x)=1-\exp (-x)$,

$$
\frac{T_{n}-n^{2} / 2}{\sqrt{n^{3} / 3}} \Rightarrow N(0,1) \text {. }
$$

- (Gumbel records) If $F(x)=1-\exp (-\exp (x))$,

$$
\frac{T_{n}-(n+1) \log n+n}{\sqrt{2 n}} \Rightarrow N(0,1) .
$$

- If $F(x)=1-(1-x)^{\gamma}$, for $0 \leq x \leq 1$,

$$
(n+1)-T_{n} \Rightarrow V_{\gamma}
$$

where $V_{\gamma}=\sum_{i=0}^{\infty} \prod_{j=0}^{i} U_{j}^{1 / \gamma}$ with $U_{j}$ 's being a sequence of i.i.d. uniform random variables.

He raised several questions in the study of partial sums of upper records; for instance of classifying all limit distributions that arise as the limits of normalised partial sums of upper records. If $X_{0}$ is bounded above and its distribution $F$ admits a density, we can give a complete answer to this question. More specifically, we show that all infinitely divisible distributions satisfying certain conditions, arise as limit distributions in this case. We derive explicit expressions for the Laplace transform of the limiting distribution and derive several distributional properties of the limiting distribution. When $X_{0}$ is not bounded above, we give sufficient conditions such that suitably normalized partial sums converge weakly to a standard normal distribution. We show that the examples worked out by Arnold are special cases of our results (Theorem 3).

In the next section, we deal with the case when the distribution is bounded above and continuous, while the unbounded case is treated in the last section. 


\section{The Bounded Case}

In this section we derive some asymptotic distributions associated with upper records when $X_{0}$ is bounded above by a constant and its distribution admits a density.

A well established method to study record values is to reduce the analysis to the case of exponential records by means of a monotone transformation. This is particularly convenient since the latter records have the same distributions as partial sums of i.i.d. exponential variables.

Let $\left\{Y_{j}: j=0,1, \ldots\right\}$ be a sequence of i.i.d. exponential random variables with parameter 1. We assume that $F$ is a continuous strictly increasing function on the set $[0, \sup (\operatorname{supp}(F))]$ where $\sup (\operatorname{supp}(F)):=\sup \{u: u \in \operatorname{supp}(F)\}$ possibly equals $+\infty)$. We define a function $g_{F}: \mathbb{R}_{+} \rightarrow \mathbb{R}_{+}$by

$$
g_{F}(x)=F^{-1}(1-\exp (-x))=F^{-1}(G(x))
$$

where $G(x)=1-\exp (-x)$ is the probability distribution function of the exponential distribution with parameter 1 . Since $F$ is continuous and strictly increasing on the set $[0, \sup (\operatorname{supp}(F))]$, so is $F^{-1}$ on $[0,1]$. Therefore, $g_{F}$ is a continuous strictly increasing function on $\mathbb{R}_{+} \rightarrow$ $[0, \sup (\operatorname{supp}(F))]$. Further, $g_{F}(0)=0$ and $g_{F}(x) \uparrow \sup (\operatorname{supp}(F))$.

It was noted by Resnick [4] that the $k^{\text {th }}$ upper record from the distribution $F$ can be represented as

$$
X_{k} \stackrel{d}{=} g_{F}\left(\sum_{j=0}^{k} Y_{j}\right) .
$$

Further, this representation holds for all joint distributions of the records, which implies that

$$
T_{n}=\sum_{k=0}^{n} X_{k} \stackrel{d}{=} \sum_{k=0}^{n} g_{F}\left(\sum_{j=0}^{k} Y_{j}\right) .
$$

For the sake of simplicity, we consider distributions having support $[0,1]$ to start with. In this case, we can easily show that $X_{n} \uparrow 1$ as $n \rightarrow \infty$.

We define

$$
h_{F}(x)=1-g_{F}(x),
$$

and for any $x \geq 0$

$$
\begin{aligned}
R_{n}(x) & :=\sum_{k=0}^{n} h_{F}\left(x+\sum_{j=0}^{k} Y_{j}\right) \\
& =\sum_{k=0}^{n}\left(1-g_{F}\left(x+\sum_{j=0}^{k} Y_{j}\right)\right) \\
& =(n+1)-\sum_{k=0}^{n} g_{F}\left(x+\sum_{j=0}^{k} Y_{j}\right) .
\end{aligned}
$$

Clearly, $R_{n}(0)=(n+1)-\sum_{k=0}^{n} X_{k}=(n+1)-T_{n}$. Notice that $\left.x+\sum_{j=0}^{k} Y_{j}\right)$ has the same distribution as the $k^{\text {th }}$ exponential upper record given that the first exceeds $x$.

We show that 
Theorem 1 For all continuous distribution functions $F$ with density $f$ on $[0,1]$ such that

$$
\int_{0}^{1} \frac{(1-u) f(u) d u}{1-F(u)}<\infty
$$

we have,

$$
(n+1)-T_{n} \Rightarrow V_{F}
$$

where $V_{F}$ is a non-negative random variable whose Laplace transform is given by

$$
\phi_{F}(-t)=E\left(\exp \left(-t V_{F}\right)\right)=\exp \left(\int_{0}^{1} \frac{(\exp (-t u)-1) f(1-u) d u}{1-F(1-u)}\right)
$$

for all $t>0$.

Proof : Let us define, for $t>0$,

$$
\psi_{t}^{(n)}(x)=E\left(\exp \left(-t R_{n}(x)\right)\right)=E\left(\exp \left(-t \sum_{k=0}^{n} h_{F}\left(x+\sum_{j=0}^{k} Y_{j}\right)\right)\right) .
$$

Since $0 \leq g_{F} \leq 1$, we have $0 \leq h_{F} \leq 1$; hence, for every fixed $x, \psi_{t}^{(n)}(x)$ is decreasing in $n$. Therefore, for each fixed $x, \psi_{t}(x):=\lim _{n \rightarrow \infty} \psi_{t}^{(n)}(x)$ exists. Further, we note that $\psi_{t}(0)=\phi_{F}(-t)$.

To prove the result, it is enough to show that $\psi_{t}(0)$, as a function of $t>0$, is the Laplace transform of a non-negative random variable whose distribution is proper; that is, without mass at infinity. We check that $\psi_{t}(0)$ is completely monotone and has the limit 1 as $t \rightarrow 0$, which suffice (by Bondesson [2], page 8 for instance). We obtain an explicit formula for $\psi_{t}(x)$ and show that under the condition $(1), \psi_{t}(0)$ indeed satisfies these two conditions.

Now, we have, by conditioning on $Y_{0}$,

$$
\begin{aligned}
\psi_{t}^{(n)}(x) & \\
= & \int_{0}^{\infty} \exp \left(-t h_{F}(x+u)\right) E\left[\exp \left(-t \sum_{k=1}^{n} h_{F}\left(x+u+\sum_{j=1}^{k} Y_{j}\right)\right)\right] \exp (-u) d u \\
= & \int_{0}^{\infty} \exp \left(-t h_{F}(x+u)\right) \psi_{t}^{(n-1)}(x+u) \exp (-u) d u \\
= & \frac{1}{\exp (-x)} \int_{x}^{\infty} \exp \left(-t h_{F}(u)\right) \psi_{t}^{(n-1)}(u) \exp (-u) d u
\end{aligned}
$$

Letting $n \rightarrow \infty$ and applying DCT on the right hand side, we have,

$$
\exp (-x) \psi_{t}(x)=\int_{x}^{\infty} \exp \left(-t h_{F}(u)\right) \psi_{t}(u) \exp (-u) d u
$$

Now, we claim that $\psi_{t}$ is the largest solution of $(3)$ in $[0,1]$. To prove this, note that if $\eta$ is any solution of $(3)$ in $[0,1]$, then we have

$$
\begin{aligned}
\psi_{t}^{(0)}(x) & =\frac{1}{\exp (-x)} \int_{x}^{\infty} \exp \left(-t h_{F}(u)\right) \exp (-u) d u \\
& \geq \frac{1}{\exp (-x)} \int_{x}^{\infty} \exp \left(-t h_{F}(u)\right) \eta(u) \exp (-u) d u \\
& =\eta(x) .
\end{aligned}
$$


By induction now it follows that $\psi_{t}^{(n)} \geq \eta$ for every $n \geq 0$. Thus, $\psi_{t} \geq \eta$.

Now, suppose that $\eta$ is any solution of $(3)$ in $[0,1]$. Clearly, the function

$$
x \rightarrow \int_{x}^{\infty} \exp \left(-t h_{F}(u)\right) \eta(u) \exp (-u) d u
$$

is continuous since the integrands are all bounded. Therefore, $\eta$ is given by the product of two continuous functions and hence is continuous. Further, once $\eta$ is continuous, the integrand in the integral $\int_{x}^{\infty} \exp \left(-t h_{F}(u)\right) \eta(u) \exp (-u) d u$ is continuous and hence the integral, as a function of $x$, is continuously differentiable. Thus, $\eta$ is given by product of two continuously differentiable functions; so it is continuously differentiable. Hence, differentiating with respect to $x$ and simplifying, we have

$$
\frac{d}{d x} \eta(x)=\eta(x)\left(1-\exp \left(-t h_{F}(x)\right)\right) .
$$

Solving this differential equation, we have,

$$
\eta(x)=K \exp \left(-\int_{x}^{\infty}\left(1-\exp \left(-t h_{F}(x)\right) d x\right)\right.
$$

where $K$ is a constant. Further, it must be the case that $0 \leq K \leq 1$, since $\eta(x) \rightarrow K$ as $x \rightarrow \infty$. Thus, all solutions of the integral equations taking values in $[0,1]$ are given by $K \exp \left(-\int_{x}^{\infty}\left(1-\exp \left(-t h_{F}(x)\right) d x\right)\right.$ where $0 \leq K \leq 1$. Now, since $\psi_{t}$ is the largest solution of the integral equation, we have,

$$
\psi_{t}(x)=\exp \left(-\int_{x}^{\infty}\left(1-\exp \left(-t h_{F}(u)\right)\right) d u\right) .
$$

Putting $x=0$, we have $\phi_{F}(-t)=\exp \left(-\int_{0}^{\infty}\left(1-\exp \left(-t h_{F}(u)\right)\right) d u\right)$. Further, $\psi_{t}(0) \neq 0$ for all $t>0$ since the integral $\int_{0}^{\infty}\left(1-\exp \left(-t h_{F}(u)\right)\right) d u$ is finite. This is because

$$
\begin{aligned}
\int_{0}^{\infty}\left(1-\exp \left(-t h_{F}(u)\right)\right) d u & \leq t \int_{0}^{\infty} h_{F}(u) d u \\
& =t \int_{0}^{\infty}\left(1-F^{-1}(1-\exp (-u))\right) d u \\
& =t \int_{0}^{1} \frac{1-F^{-1}(u)}{1-u} d u \\
& =t \int_{0}^{1} \frac{(1-u) f(u)}{1-F(u)} d u \\
& <\infty .
\end{aligned}
$$

It is fairly straightforward to show that $\psi_{t}(0)$ is completely monotone; that is, $(-1)^{n} \frac{d^{n}}{d t^{n}} \psi_{t}(0) \geq$ 0. Differentiating under the sign of the integral, we have

$$
\frac{d}{d t} \psi_{t}(0)=-\psi_{t}(0) \int_{0}^{\infty} \exp \left(-t h_{F}(u)\right) h_{F}(u) d u .
$$

Note that this is permissible when the integral $\int_{0}^{\infty} \exp \left(-t h_{F}(u)\right) h_{F}(u) d u<\infty$. Using induction, one can easily verify that $(-1)^{n} \frac{d^{n}}{d t^{n}} \psi_{t}(0) \geq 0$. 
Finally, we show that $\psi_{t}(0) \rightarrow 1$ as $t \rightarrow 0$. Clearly, as $t \rightarrow 0$, we have $1-\exp \left(-t h_{F}(u)\right) \rightarrow 0$. For $t<1$, we have $1-\exp \left(-t h_{F}(u)\right) \leq h_{F}(u)$ and by the above calculations, $\int_{0}^{\infty} h_{F}(u) d u<\infty$. Therefore, by DCT, we obtain $\psi_{t}(0) \rightarrow 1$ as $t \rightarrow 0$. This proves the result.

Remark Note that $0 \leq X_{n} \leq 1$ for all $n \geq 0$. This implies that, $(n+1)-T_{n}=\sum_{j=0}^{n}\left(1-X_{j}\right)$ is a non-decreasing sequence. Therefore, $\sum_{j=0}^{n}\left(1-X_{j}\right)=\lim _{n \rightarrow \infty}(n+1)-T_{n}$ converges but may possibly take value $+\infty$ with positive probability. However, under our assumption (1), since $(n+1)-T_{n}$ converges weakly to a proper random variable (that is, without mass at infinity), we have

$$
\sum_{n=0}^{\infty}\left(1-X_{n}\right) \stackrel{d}{=} V_{F}
$$

Further, the condition $\int_{0}^{1} \frac{1-F^{-1}(u)}{1-u} d u<\infty$, equivalent to (1) when the density exists, is enough to guarantee the convergence even when the density may not exist, in which case the Laplace transform of $V_{F}$ is given by

$$
\phi_{F}(-t)=\exp \left(-\int_{0}^{\infty}\left(1-\exp \left(-t h_{F}(u)\right)\right) d u\right) .
$$

Now, if the integral condition in (1) does not hold, that is,

$$
\int_{0}^{1} \frac{(1-u) f(u) d u}{1-F(u)}=\infty
$$

we show that $(n+1)-T_{n} \rightarrow \infty$ almost surely.

Lemma 1 If (6) holds,

$$
(n+1)-T_{n} \rightarrow \infty \text { almost surely }
$$

Proof : In view of the above remark, we need to prove that $(n+1)-T_{n} \Rightarrow \infty$. For that, it is sufficient to show that the only bounded solution admitted by equation (4) is 0 . Now, if $\eta$ is any solution of (4), we have

$$
\frac{d}{d x}\left(\eta(x) \exp \left(-\int_{0}^{x}\left(1-\exp \left(-t h_{F}(u)\right)\right) d u\right)\right)=0 .
$$

Suppose that $\eta(0)=K$ where $0 \leq K \leq 1$. Then, we have

$$
\eta(x)=K \exp \left(\int_{0}^{x}\left(1-\exp \left(-t h_{F}(u)\right)\right) d u\right) \text { for } x>0 .
$$

Now, we have $\int_{0}^{x}\left(1-\exp \left(-t h_{F}(u)\right)\right) d u \rightarrow \int_{0}^{\infty}\left(1-\exp \left(-t h_{F}(u)\right)\right) d u$ as $x \rightarrow \infty$. Further, we have that

$$
\begin{aligned}
\int_{0}^{\infty} & \left(1-\exp \left(-t h_{F}(u)\right)\right) d u \\
\quad= & \int_{0}^{1} \frac{(1-\exp (-t(1-u))) f(u)}{1-F(u)} d u \\
\geq & C_{t} \int_{0}^{1} \frac{(1-u) f(u)}{1-F(u)} d u \\
\quad= &
\end{aligned}
$$


where $C_{t}>0$ is a constant depending only on $t$. Thus, if $K \neq 0$, we must have $\eta(x) \uparrow \infty$ as $x \rightarrow \infty$. This completes the proof

This result can easily be generalised to the case when $\operatorname{supp}(F)=[0, c]$ where $c \neq 1$. Clearly scaling the distribution by $1 / c$, we can reduce the situation to a distribution on $[0,1]$ and apply the results just proved above. Then, scaling back, we can obtain the following corollary. In this case too, the existence of the density is only to allow a neat expression for the Laplace transform.

Corollary 1.1 Suppose that $F$ is a distribution function with density $f$ on $[0, c]$. Then,

$$
(n+1) c-T_{n} \Rightarrow V_{F}(c)
$$

if and only if

$$
\int_{0}^{c} \frac{(c-u) f(u) d u}{1-F(u)}<\infty
$$

where $V_{F}(c)$ is a non-negative random variable whose moment generating function is given by

$$
\phi_{F}(-t)=E\left(\exp \left(-t V_{F}(c)\right)\right)=\exp \left(\int_{0}^{c} \frac{(\exp (-t u)-1) f(c-u) d u}{1-F(c-u)}\right)
$$

for all $t>0$.

More generally, if $\int_{0}^{c} \frac{\left(c-F^{-1}(u) d u\right.}{1-u}<\infty$, the limiting distribution exists and its Laplace transform is given by

$$
\phi_{F}(-t)=\exp \left(-\int_{0}^{\infty}\left(1-\exp \left(-t h_{F}(u)\right)\right) d u\right)
$$

where $h_{F}(u)$ is now defined as $c-g_{F}(u)$.

\section{Properties of the Asymptotic Distribution}

In this section, we derive the properties of the asymptotic distribution obtained in the last section and describe the class of distributions which can originate as the limiting distribution.

Proposition 3.1 If $F$ has support on $[0, c]$ and admits a density $f$ such that

$$
\int_{0}^{c}(c-u) f(u) d u / F(u)<\infty
$$

then the distribution of $V_{F}(c)=\sum_{n=0}^{\infty}\left(c-X_{n}\right)$ is infinitely divisible.

Proof : Clearly the Laplace transform of the infinite sum can be written as

$$
E\left(\exp \left(-t V_{F}(c)\right)\right)=\exp \left(\int_{0}^{c} \frac{(\exp (-t u)-1) g_{c}(u) d u}{G_{c}(u)}\right)
$$

where $g_{c}(u)=f(c-u)$ and $G_{c}(u)=\int_{0}^{u} g_{c}(v) d v$. 
From Bondesson [2], a random variable taking values in $\mathbb{R}_{+}$, is infinitely divisible (i.d.) if and only if the moment generating function can be expressed as

$$
m(t)=\exp \left(-a t+\int_{(0, \infty)}(\exp (-t u)-1) L(d u)\right)
$$

where $t>0, a \geq 0$ and $L$, the Levy measure, is non-negative and satisfies

$$
\int_{(0, \infty)} \min (1, u) L(d u)<\infty .
$$

Setting $a=0$ and $L(d u)=1_{(0, c)}(u) g_{c}(u) d u / G_{c}(u)$, the moment generating function of $V_{F}(c)$ can be written in the above form. Also, we have

$$
\int_{0}^{\infty} \min (1, u) L(d u) \leq \int_{0}^{c} \frac{u g_{c}(u)}{G_{c}(u)} d u=\int_{0}^{c} \frac{(c-u) f(u)}{F(u)}<\infty .
$$

This completes the proof.

Clearly, we have that the Levy measure $L$ admits a density which is given by $l(y)=$ $g_{c}(y) / G_{c}(y)$ for $0 \leq y \leq c$. Now, conversely suppose that we are given any infinitely divisible distribution on $[0, \infty)$ with a Levy measure which admits a density $l(y)$ on $[0, c]$ for some $c>0$. We further assume that the mass of the Levy measure is infinite, i.e.,

$$
\int_{0}^{c} l(y) d y=\infty
$$

Further, it must be the case that

$$
\int_{0}^{c} \min (1, y) l(y) d y<\infty
$$

We first note that (9) implies $\int_{x}^{c} l(y) d y<\infty$ for all $0<x<c$, for

$$
\int_{0}^{c} \min (1, y) l(y) d y \geq \int_{x}^{c} \min (1, y) l(y) d y \geq \min (1, x) \int_{x}^{c} l(y) d y .
$$

Now, define a representation for $g_{c}$ in the following way

$$
g_{c}(x)=l(x) \exp \left(-\int_{x}^{c} l(y) d y\right)
$$

for $0 \leq x \leq c$. Then we have that,

$$
G_{c}(x)=\exp \left(-\int_{x}^{c} l(y) d y\right)
$$

It is clear from the above definition that we have,

$$
\int_{0}^{c} \frac{(c-u) f(u)}{1-F(u)} d u<\infty
$$

where $f(u)=g_{c}(c-u)$ for $0 \leq u \leq c$. Therefore, we must have that $(n+1) c-T_{n}$ converges almost surely to a positive random variable $K$. It is clear that the Laplace transform of $K$ is same as that of the given infinitely divisible distribution. Therefore, we have the following characterisation result of all possible limit laws which can arise in this way. 
Theorem 2 For any infinitely divisible random variable $K(c)$ on $[0, \infty)$ such that the Levy measure has a density $l(y)$ on $[0, c]$ such that $\int_{0}^{c} l(y) d y=\infty$, there exists a distribution $F$ with density on $[0, c]$ such that

$$
(n+1) c-T_{n} \rightarrow K \quad \text { almost surely }
$$

where $T_{n}$ is the partial sum of first $(n+1)$ records from the distribution $F$. Moreover, the density of $F$ is given by $f(u)=g_{c}(c-u)$ where $g_{c}$ is given above.

The other natural question that we can ask here is: under what conditions on $F$ will the distribution of $Y$ belong to specified subclasses of the class of i.d. laws. For a comprehensive description of interesting subclasses of i.d. laws, see Bondesson ([2]).

The first and relatively simple characterization is for the class $\mathcal{L}$, the class of self decomposable laws, consisting of distributions of those random variables $X$ for which for every $c \in(0,1]$, there exists a random variable $\epsilon_{c}$ independent of $X$ such that $X \stackrel{d}{=} c X+\epsilon_{c}$.

Observing that the Levy density of $Y$ is given by $g_{c}(y) / G_{c}(y)$, we obtain the following:

Proposition 3.2 If the density function $f$ is such that $u g_{c}(u) / G_{c}(u)=u f(c-u) /(1-F(c-u))$ is decreasing in $u$ and $\int_{0}^{c}\left(u g_{c}(u) / G_{c}(u)\right) d u<\infty$, then $V_{F}(c)$ is self-decomposable.

The proof is straightforward from Bondesson ([2], page 18). Note that the Levy density $l(y)=\beta y^{-1} \exp (-t y) 1_{\{y>0\}}$ characterises the Gamma distribution $\mathbf{G}(\beta, t)$ where $\beta, t>0$. This will arise as the distribution of $V_{F}(c)$ if

$$
F(x)=\exp \left(-\beta \int_{x}^{\infty} y^{-1} \exp (-t y) d y\right) .
$$

The subclass $\mathcal{T}_{2}$ of i.d. laws comprises of generalized mixtures of exponential distributions arising as weak limits of mixtures of exponentials. This class is characterised by complete monotonicity of the Levy density. Using this, $V_{F}(c)$ belongs to $\mathcal{T}_{2}$ if $-\log \left(G_{c}(\cdot)\right)$ is completely monotone.

A subclass of both $\mathcal{L}$ and $\mathcal{T}_{2}$ is the class $\mathcal{T}$ consisting of Generalized Gamma convolutions defined as weak limits of finite convolutions of Gamma distributions. By Theorem 3.1.1 of [2], a distribution $G$ which gives full mass to the set of nonnegative real numbers belongs to the class $\mathcal{T}$ if and only if its Levy density $l(\cdot)$ satisfies

$$
y l(y)=\int_{0}^{\infty} \exp (-y t) U(d t)
$$

for all $y>0$, and for some measure $U$ on $(0, \infty)$ with $\int_{1}^{0}|\log t| U(d t)<\infty$ and $\int_{\infty}^{1} t^{-1} U(d t)<\infty$. Equivalently, if $y l(y)$ is completely monotone on $(0, \infty)$.

As example of this relation, we may mention the stable distributions when $l(y) \propto y^{\alpha-1}$ for $y>0$ where $0<\alpha<1$. These arise from the case $G_{1}(x)=\exp \left(-x^{-\alpha}\right), x>0$.

When does the distribution of $V_{F}(c)$ admit a density? Using the infinite divisibility, we may obtain answers to this question. 
Proposition 3.3 The random variable $V_{F}(c)$ is absolutely continuous with respect to the Lebesgue measure.

Proof : Hudson and Tucker [3] obtained sufficient conditions for an infinitely divisible distribution to be equivalent to the Lebesgue measure. Using Theorem 1 of Hudson and Tucker [3], it is enough to verify that the Levy measure is absolutely continuous with respect to the Lebesgue measure, has infinite mass and finite first moment.

In our case, we have the Levy measure as $g_{c}(x) / G_{c}(x) 1_{(0, c)}(x) d x$ and satisfies the properties of absolute continuity and the infinite mass (see (8)). Further, we have $\int_{0}^{c} u g_{c}(u) d u / G_{c}(u) \leq$ $K \int_{0}^{c} \min (1, u) g_{c}(u) d u / G_{c}(u)<\infty$ where $K>0$ is a constant; hence the first moment is finite. This proves the result.

\section{Unbounded Case}

In this section, we look at the general case when the support of $F$ is not bounded. From Resnick [4], it is clear that we need to impose conditions for convergence. We assume that

$$
g_{F} \text { is twice differentiable on } \mathbb{R}_{+}
$$

and there exists constants $C_{1}$ and $C_{2}$ such that

$$
\left|g_{F}^{\prime \prime}(x)\right|^{2} \leq C_{1}\left(\left|g_{F}^{\prime \prime}\left(x_{1}\right)\right|^{2}+\left|g_{F}^{\prime \prime}\left(x_{2}\right)\right|^{2}\right)
$$

whenever $0<x_{1}<x<x_{2}<\infty$ and for all $k \geq 0$,

$$
E\left(\left|g_{F}^{\prime \prime}\left(\sum_{j=0}^{k} Y_{j}\right)\right|^{2}\right) \leq C_{2}\left|g_{F}^{\prime \prime}(k+1)\right|^{2}
$$

where $\left\{Y_{j}: j \geq 0\right\}$ is a sequence of i.i.d. exponential random variable.

Now, we define $a_{n}=g_{F}^{\prime}(n)$ and $b_{j}(n)=\sum_{k=j}^{n} a_{k+1}$ for $0 \leq j \leq n$ and $c_{n}^{2}=\sum_{j=0}^{n}\left(b_{j}(n)\right)^{2}$. Further, denote $d_{n}=\left|g_{F}^{\prime \prime}(n+1)\right|$. We impose two technical conditions on the sequences; namely,

$$
\frac{1}{c_{n}} \sum_{j=0}^{n}(j+1) d_{j} \rightarrow 0 \text { as } n \rightarrow \infty
$$

and

$$
\frac{1}{c_{n}} \max _{0 \leq j \leq n}\left|b_{j}(n)\right| \rightarrow 0 \text { as } n \rightarrow \infty .
$$

Under these two technical conditions, we are able to obtain asymptotic normality for the sequence of partial sums of records.

Theorem 3 For a general distribution $F$ for which the conditions (11), (12), (13), (14) and (15) are satisfied,

$$
\frac{\sum_{k=0}^{n}\left(X_{k}-g_{F}(k+1)\right)}{c_{n}} \Rightarrow N(0,1)
$$


Proof : We will use the representation of the records in terms of exponential random variables. Using Taylor Series expansion for $g_{F}$, we have

$$
\begin{aligned}
& \sum_{k=0}^{n}\left(X_{k}-g_{F}(k+1)\right) \\
& \quad=\sum_{k=0}^{n}\left[g_{F}\left(\sum_{j=0}^{k} Y_{j}\right)-g_{F}(k+1)\right] \\
& \quad=\sum_{k=0}^{n} g_{F}^{\prime}(k+1) \sum_{j=0}^{k}\left(Y_{j}-1\right)+\frac{1}{2} \sum_{k=0}^{n}\left(\sum_{j=0}^{k}\left(Y_{j}-1\right)\right)^{2} g_{F}^{\prime \prime}\left(S_{k}^{\star}\right) \\
& =T_{n}+E_{n}
\end{aligned}
$$

where $T_{n}=\sum_{k=0}^{n} a_{k+1} \sum_{j=0}^{k}\left(Y_{j}-1\right)=\sum_{j=0}^{n}\left(Y_{j}-1\right) \sum_{k=j}^{n} a_{k+1}=\sum_{j=0}^{n}\left(Y_{j}-1\right) b_{j}(n)$ and $E_{n}=\sum_{k=0}^{n}\left(\sum_{j=0}^{k}\left(Y_{j}-1\right)\right)^{2} g_{F}^{\prime \prime}\left(S_{k}^{\star}\right) / 2$ where $S_{k}^{\star}$ is a point in between $\sum_{j=0}^{k} Y_{j}$ and $k+1$.

First we show that $T_{n} / c_{n}$ converges to a normal distribution weakly. Indeed, we can derive the characteristic function of the above sum. For any $t \in \mathbb{R}$, we have

$$
\begin{aligned}
& E\left(\exp \left(i t T_{n} / c_{n}\right)\right) \\
& =\exp \left(-i t / c_{n} \sum_{j=0}^{n} b_{j}(n)\right) \prod_{j=0}^{n} E\left(\exp \left(i t b_{j}(n) Y_{j} / c_{n}\right)\right) \\
& \quad=\exp \left(-i t / c_{n} \sum_{j=0}^{n} b_{j}(n)\right) \prod_{j=0}^{n} \frac{1}{1-i t b_{j}(n) / c_{n}} .
\end{aligned}
$$

Now, taking logarithm, we have for all $t$ with $|t|<c_{n} / \max _{j}\left|b_{j}(n)\right|$,

$$
\begin{aligned}
& \left|\log E\left(\exp \left(i t T_{n} / c_{n}\right)\right)-t^{2} / 2\right| \\
& \quad=\left|-\sum_{j=0}^{n} \frac{i t b_{j}(n)}{c_{n}}-\frac{t^{2}}{2}-\sum_{j=0}^{n} \log \left(1-i t b_{j}(n) / c_{n}\right)\right| \\
& \leq \frac{|t|^{3}}{3 c_{n}^{3}} \sum_{j=0}^{n}\left|b_{j}(n)\right|^{3} \leq \frac{|t|^{3}}{3 c_{n}^{3}} \max _{j}\left|b_{j}(n)\right| \sum_{j=0}^{n}\left(b_{j}(n)\right)^{2} \\
& =\frac{|t|^{3}}{3 c_{n}} \max _{j}\left|b_{j}(n)\right|
\end{aligned}
$$

This implies, by (15), that for all $t \in \mathbb{R}, E\left(\exp \left(i t T_{n} / c_{n}\right)\right) \rightarrow \exp \left(-t^{2} / 2\right)$ as $n \rightarrow \infty$.

Now, we are to show that $E_{n} / c_{n} \rightarrow 0$ in probability. For that, it is enough to show that $E\left(\left|E_{n} / c_{n}\right|\right) \rightarrow 0$ as $n \rightarrow \infty$. Clearly,

$$
E\left(\left|E_{n} / c_{n}\right|\right) \leq \sum_{j=0}^{n} E\left(\left(\sum_{j=0}^{k}\left(Y_{j}-1\right)\right)^{2}\left|g_{F}^{\prime \prime}\left(S_{k}^{\star}\right)\right|\right) /\left(2 c_{n}\right) .
$$

Now, using Cauchy-Schwartz inequality, we have

$$
E\left(\left(\sum_{j=0}^{k}\left(Y_{j}-1\right)\right)^{2}\left|g_{F}^{\prime \prime}\left(S_{k}^{\star}\right)\right|\right) \leq\left[E\left(\sum_{j=0}^{k}\left(Y_{j}-1\right)\right)^{4}\right]^{1 / 2}\left[E\left(g_{F}^{\prime \prime}\left(S_{k}^{\star}\right)\right)^{2}\right]^{1 / 2} .
$$


Since, $Y_{j}$ 's are i.i.d. exponential random variables, it is easy to show that

$$
E\left(\sum_{j=0}^{k}\left(Y_{j}-1\right)\right)^{4} \leq C_{3}(k+1)^{2}
$$

for some constant $C_{3}>0$. Now, since $S_{k}^{\star}$ lies in between $(k+1)$ and $\sum_{j=0}^{k} Y_{j}$, we have from condition (12) and (13),

$$
\begin{aligned}
E\left(g_{F}^{\prime \prime}\left(S_{k}^{\star}\right)\right)^{2} & \leq C_{1}\left[\left(g_{F}^{\prime \prime}(k+1)\right)^{2}+E\left(g_{F}^{\prime \prime}\left(\sum_{j=0}^{k} Y_{j}\right)\right)^{2}\right] \\
& \leq C_{1}\left[\left(g_{F}^{\prime \prime}(k+1)\right)^{2}+C_{2}\left(g_{F}^{\prime \prime}(k+1)\right)^{2}\right] \\
& =C_{1}\left(d_{k}\right)^{2}\left(1+C_{2}\right) .
\end{aligned}
$$

Thus, we have from (16) and (17),

$$
\begin{aligned}
& E\left(E_{n} / c_{n}\right) \\
& \leq \frac{1}{c_{n}} \sum_{j=0}^{n}\left(C_{3}(k+1)^{2}\right)^{1 / 2}\left(C_{1}\left(1+C_{2}\right) d_{k}^{2}\right)^{1 / 2} \\
& =\frac{\left(C_{1} C_{3}\left(1+C_{2}\right)\right)^{1 / 2}}{c_{n}} \sum_{j=0}^{n}(k+1) d_{k} \rightarrow 0
\end{aligned}
$$

as $n \rightarrow \infty$. This completes the proof.

The conditions imposed on the function $g_{F}$ are reasonably general. We show that two important functions satisfy them, so that the specific cases considered by Arnold [1] mentioned at the beginning of Section 1 are included. First, we consider Gumbel records.

Proposition 4.1 The function $g_{F}(x)=\log (x)$ satisfies the conditions (11) - (15).

Proof : Clearly $g_{F}^{\prime}(x)=1 / x$ and $g_{F}^{\prime \prime}(x)=-1 / x^{2}$. Thus, $a_{n}=1 / n$ and $d_{n}=1 /(n+1)^{2}$. Now, $b_{j}(n)=\sum_{k=j}^{n} a_{k+1}=\sum_{k=j+1}^{n+1} k^{-1}$. Easy calculations imply that $c_{n}^{2} /(2 n) \rightarrow 1$ as $n \rightarrow \infty$. Further,

$$
\left(g_{F} \prime \prime(x)\right)^{2}=\frac{1}{x^{4}} \leq \frac{1}{x_{1}^{4}}+\frac{1}{x_{2}^{4}}=\left(g_{F} \prime \prime\left(x_{1}\right)\right)^{2}+\left(g_{F} \prime \prime\left(x_{2}\right)\right)^{2}
$$

whenever $0<x_{1}<x<x_{2}$. Now,

$$
\begin{aligned}
E\left(\left(g_{F} \prime \prime\left(\sum_{j=0}^{k} Y_{j}\right)\right)^{2}\right) & =\int_{0}^{\infty} \frac{1}{\Gamma(k+1)} \frac{\exp (-x) x^{k}}{x^{4}} d x \\
& =\frac{1}{k(k-1)(k-2)(k-3)} \\
& \leq \frac{C_{4}}{k^{4}}
\end{aligned}
$$


for some constant $C_{4}>0$. Finally,

$$
\frac{1}{c_{n}} \sum_{k=0}^{n}(k+1) d_{k}=\frac{1}{c_{n}} \sum_{k=0}^{n} \frac{1}{k+1} \leq \frac{C_{5} \log n}{\sqrt{n}} \rightarrow 0
$$

as $n \rightarrow \infty$ where $C_{5}>0$ is a constant. Also, it is easy to verify that $\max _{j}\left|b_{j}(n)\right|=b_{0}(n)=$ $\sum_{1}^{n+1} k^{-1}<\left\lceil\log _{2} n\right\rceil+1$, so that (15) is also satisfied. This completes the proof.

Finally we prove that also the more general class of Weibull distributions containing the Exponential is covered. In the Proposition below, the choice $\delta=1$ reduces to the Exponential case.

Proposition 4.2 The function $g_{F}(x)=x^{\delta}$, where $\delta>0$, satisfies (11) - (15).

Proof : Here we have $g_{F}^{\prime}(x)=\delta x^{\delta-1}$ and $g_{F}^{\prime \prime}(x)=\delta(\delta-1) x^{\delta-2}$ (which is 0 when $\delta=1$ ), $a_{n}=\delta n^{\delta-1}$ and $d_{n}=\delta|\delta-1|(n+1)^{\delta-2}$ (with $d_{n}=0$ when $\left.\delta=1\right)$. Now, $b_{j}(n)=$ $\sum_{k=j}^{n} a_{k+1}=\delta \sum_{k=j+1}^{n+1} k^{\delta-1}$. Again, easy calculations show that $c_{n}^{2} /(n+1)^{1+2 \delta} \rightarrow \lambda_{\delta}$ where $\lambda_{\delta}=(1-2 /(1+\delta)+1 /(1+2 \delta))$.

Now, for $\delta=1$, the conditions (12) and (13) are trivially satified. For $\delta \neq 1$, we have

$$
\begin{aligned}
\left(g_{F}^{\prime \prime}(x)\right)^{2} & =\delta^{2}(\delta-1)^{2} x^{2 \delta-2} \leq \delta^{2}(\delta-1)^{2} x_{1}^{2 \delta-2}+\delta^{2}(\delta-1)^{2} x_{2}^{2 \delta-2} \\
& =\left(g_{F}^{\prime \prime}\left(x_{1}\right)\right)^{2}+\left(g_{F}^{\prime \prime}\left(x_{2}\right)\right)^{2}
\end{aligned}
$$

whenever $0<x_{1}<x<x_{2}<\infty$. Now,

$$
E\left(g_{F}^{\prime \prime}\left(\sum_{j=0}^{k} Y_{j}\right)\right)^{2}=\delta^{2}(\delta-1)^{2} \Gamma((k+1)+2(\delta-2)) / \Gamma(k+1) .
$$

As $k \rightarrow \infty$, we have $\Gamma((k+1)+2(\delta-2)) /\left(k^{2(\delta-2)} \Gamma(k+1)\right) \rightarrow 1$. Thus, we have, for some $C_{7}>0$,

$$
E\left(g_{F}^{\prime \prime}\left(\sum_{j=0}^{k} Y_{j}\right)\right)^{2} \leq C_{7}\left(g_{F}^{\prime \prime}(k+1)\right)^{2} .
$$

When $\delta=1$, the condition (14) is trivially satisfied. For $\delta \neq 1$,

$$
\begin{aligned}
\frac{1}{c_{n}} \sum_{k=0}^{n}(k+1) d_{k} & \leq \frac{C_{8}}{n^{\delta+1 / 2}} \sum_{k=0}^{n} k^{\delta-1} \\
& \leq \frac{C_{9} n^{\delta}}{n^{\delta+1 / 2}} \rightarrow 0
\end{aligned}
$$

as $n \rightarrow \infty$ where $C_{8}>0$ and $C_{9}>0$ are constants. Finally, $\max _{j}\left|b_{j}(n)\right|=b_{0}(n) \leq C_{10}(n+1)^{\delta}$ whence

$$
\left.\frac{1}{c_{n}} \max _{j} \mid b_{j}(n)\right) \mid \leq \frac{C_{11}}{(n+1)^{1 / 2}} \rightarrow 0
$$

as $n \rightarrow \infty$ where $C_{10}>0$ and $C_{11}>0$ are constants. This completes the proof.

Acknowledgement. We are grateful to Professor Barry Arnold for providing us with a copy of his work. Anish Sarkar thanks Prof. L. R. Fontes for many suggestions and acknowledges the support received from the Brasilian Academy of Sciences, the Indian National Science Academy and Instituto de Matemática e Estatística (IME), the Universidade de São Paulo. 


\section{References}

[1] B. C. Arnold The asymtotic distributions of sums of records. Extremes, 1(3): 351 - 363, 1999.

[2] L. Bondesson. Generalized Gamma Convolutions and Related Classes of Densities. Springer-Verlag, Lecture Notes in Statistics \# 76, 1992.

[3] W. N. Hudson and H. G. Tucker. Equivalence of infinitely divisible distributions. Annals of Probability, 3: 70 - 79, 1975.

[4] S. I. Resnick. Limit laws for record values. Stochastic Processes and Their Applications, 1: $67-82,1964$. 